\title{
Adding sound to lipread lists: The effects on serial recall of adding an auditory pulse train and a pure tone to silently lipread lists
}

\author{
RUTH CAMPBELL and JEANETTE GARWOOD \\ University of Oxford, Oxford, England \\ and \\ STUART ROSEN \\ University College London, London, England
}

\begin{abstract}
These investigations examined subjects' serial recall of lipread digit lists accompanied by an auditory pulse train. The pulse train indicated the pitch of voiced speech (buzz-speech) of the seen speaker as she was speaking. As a purely auditory signal, it could not support item identification. Such buzz-speech recall was compared with silent lipread list recall and with the recall of buzz-speech lists to which a pure tone had been added (buzz-and-beep lists). No significant difference in overall accuracy of recall emerged for the three types of lipread list; however, there were significant differences in the shape of the serial recall function for the three list types. Recency characterized the silent and the buzz-speech lists, and these lists differed in their varying susceptibilities to a range of speechlike suffixes. By contrast, adding a pure tone to a buzz-speech list (buzz-and-beep) produced little recency and no further recall loss as a function of suffix type. We discuss these effects with reference to the contrast between sensory-similarity and speechlikeness accounts of auditory recency and suffix effects. Sensory similarity accounts cannot capture the effects reported here, but processing in a speech mode (buzz-and-beep) need not always lead to recency effects like those resulting from clearly heard or lipread lists.
\end{abstract}

One of the most robust and reliable phenomena in immediate short-term memory is the modality-specific recency effect. The last item of a heard list is better remembered than the last item of a list that is read silently. This last-item advantage is lost when the heard list is followed by a subsequent speech sound; this is the recencyspecific suffix effect (Crowder \& Morton, 1969).

However, lists do not have to be spoken for such modality effects to occur. Silently lipread lists also show recency compared with written lists, and this recency, like auditory recency, is lost when the lipread list is followed by a spoken suffix (Campbell $\&$ Dodd, 1980). Lipread and heard recency and suffix effects seem to share a common source; thus, for both heard and silently lipread lists, only a speechlike suffix (lipread or heard) reduces recency; a written suffix has no effect, nor does a suffix that involves tongue movements that are not related to speech (Campbell \& Dodd, 1982; Gathercole, 1987; Spoehr \& Corin, 1978). ${ }^{1}$

We gratefully acknowledge the technical help of University College London's Audiovisual Unit in preparing some of the material used in this study. This work was supported by an Oxford University pumppriming grant. We are most grateful to Tom Ayres for directing us to Routh and Lifschutz's (1975) article. Address correspondence to Ruth Campbell, Department of Experimental Psychology, University of Oxford, South Parks Road, Oxford OX1 3UD, England.
Underlying these experiments is the idea that recency in the immediate recall of simple, serially ordered lists reflects access to a resilient immediate memory trace, the nature of which can be inferred by the susceptibility of recency to suffixes of different kinds. Classical work by Morton, Crowder, and others (see Crowder, 1983, for a review) made clear that this trace is precategorical; that is, it is not subject to lexical constraints. Moreover, the finding that lipread recency is reduced by auditory and lipread suffixes, but not by a graphic suffix or by a suffix formed by the nonspeech lip movement of tongue protrusion, suggests that the trace that gives rise to lipread recency has much in common with heard and lipread speech and little in common with nonspeech lip movements or with written material.

What more can be said about the nature of this lipread trace? Precisely how does it resemble and how does it differ from one that is heard? In one study (Campbell \& Dodd, 1984, Experiment 3) it was shown that recency from silent lipreading is susceptible to overwriting from a number of different speechlike suffixes; in particular, a heard man's voice and a heard woman's voice had equally deleterious effects on the recall of the last item of a lipread list that was seen to be spoken by a woman. By contrast, an auditory list is more specifically affected by the voice of the suffix; a man's voice speaking a suffix interferes more with the recall of a list spoken by a man 
than does a woman's voice (Greenberg \& Engle, 1983; Morton, Crowder, \& Prussin, 1971).

It appears therefore, that the lipread trace, unlike the auditory trace, does not contain presumed auditory characteristics of voice pitch and timbre, although these could be readily inferred from viewing the speaker; we all know what a woman's speaking voice should sound like and what a man's should sound like. For lipread lists, recency and suffix effects appear to reflect the use of a somewhat more abstractly coded trace, one that could be termed phonetic, a term that is neutral with respect to modality of the speech event (see Campbell, 1987; also Crowder, 1983; Morton, Marcus, \& Ottley, 1981). For lists that are heard, a possibly independent source of recency and suffix effects may be the sensory similarity of list and suffix. Thus, in Morton et al.'s (1971) experiment, two effects may have been operating: a phonetic recency and suffix effect, which is relatively insensitive to the surface characteristics of the heard list, and a sensory-specific effect, which gives rise to differential suffix effects for heard voice differences between list and suffix.

In this study, we explored the immediate recall of lipread lists when lipreading was accompanied by an auditory pulse train that was driven by the opening and closing of the speaker's vocal folds. In studies that simulated the effects of cochlear implantation for total deafness, Rosen, Fourcin, and Moore (1981) used a neck-mounted laryngograph to respond to a speaker's vocal fold activity. Each vocal fold closure caused the laryngograph to generate a pulse that was presented to a listener through headphones or loudspeakers. By this means, the voice pitch of the speaker was communicated to the listener. Rosen et al. demonstrated the utility of such variable-pitch buzzspeech accompanying lipreading, reporting up to a twofold increase in speed in connected discourse tracking (i.e., in accurately repeating a passage of lipread text aloud) for passages of text accompanied by buzz.

The initial auditory impression of this manipulation is of lipreading while hearing an interrupted stream of buzzes. However, after some exposure, this impression can change, and the perception can be similar to that of listening to noisy speech, rather than to a buzzed accompaniment to lipreading. In other words, an integrated speech percept can emerge from these disparate inputs. Such integration not only has the perceptual quality of heard speech, but lipreading comprehension is improved by it, just as it is by low levels of auditory input.

This effect is particularly intriguing since the acoustic pulse train is lacking in many of the natural qualities of human speech. The pulse train can generate no voiceless sounds, and it produces an unvarying spectral envelope (it has some features in common with natural voiced speech, e.g., its periodicity). When listening but not viewing, the listener hears only an intermittent stream of buzzes, of variable pitch and similar loudness; the effect is similar to hearing someone speaking on the other side of a solid wall. One may have the impression that speech is being uttered, but the language being spoken and the nature of the speech sounds are quite unidentifiable. What remains is the pitch contour of the speech, indicating prosodic contour.

How then is such seen buzz-speech recalled? Does the advantage of the auditory pulse train in tracking a seen speaker reading unfamiliar passages from murder mysteries (Rosen et al.'s, 1981, experiment) extend to the task of recalling lists of numbers? Remembering lists of silently presented, lipread numbers is a task at which experimental subjects (typically undergraduates) achieve asymptotic performance after a few minutes of familiarization. By contrast, the tracking of silently spoken text requires some practice and, even then, is susceptible to many "derailments." In the recall task, the number of (lexical) items (single digits, prescribed letter names) is finite, few, and known in advance to the viewer. In the tracking task, there are no such constraints.

Does the serial recall curve for buzz-speech resemble that for silent lipread speech? If, like silent lipread lists, such buzz-speech lists show recency, is buzz-speech susceptible to the same sorts of suffix effect as is silent lipread speech, or, rather, since the subjective impression is of hearing speech, are there distinguishable suffix effects, depending on the similarity of list and suffix? We expect this latter pattern for clear auditory presentation, but it has not been shown to occur for lipread lists.

\section{EXPERIMENT 1}

The first experiment reported here examined the serial recall of buzz-speech lists of digits and compared it with that for silently lipread lists. Four list conditions were examined. In the no-suffix condition, nine single-digit number lists were presented by silent lipreading or by lipreading accompanied by an auditory pulse train and were to be recalled in strict serial order. The other three conditions included a suffix that subjects were instructed to ignore. In all cases, this suffix was seen to be the word "go" being spoken. In one condition "go" was silent, in another it was in buzz-speech, and in the third condition it was clearly heard as well as seen.

If buzz-speech lists show recency, then the effects of the different suffix types would indicate something concerning the resilient memory trace for such partial speech stimuli, where the auditory information can be processed as speech only when the speaker is seen as well as heard. If a buzzspeech suffix has the most marked effect, then it would seem that sensory structures are important in the construction of the trace. However, if a fully auditorilly specified speech suffix has the more marked effect, then the resilient trace would seem to be organized in speechlike terms that are somewhat independent of stimulus characteristics.

\section{Method}

Subjects. Twenty-two undergraduate students of speech science at the National Hospitals' College of Speech Science, London, volunteered for the 30-min experiment. They were aged between 18 and 25 years; none had any formal training in speech reading at this 
stage, and they were naive as to the nature of the experimental enquiry. Twelve of these subjects were assigned to the buzz-speech condition and 10 to the silent lipreading condition. Groups were tested concurrently in different rooms.

Stimulus materials. A single videotape was prepared, which contained all list types. Two videotapes were produced from this; one for the silent lipreading condition and one for the buzz-speech condition. The general form of the videotape was as follows: a single speaker (J.G.) was recorded as she spoke aloud lists of single digits. J.G. wore a throat-mounted electrolaryngograph, which indicated vocal fold closures by the output of a constant-amplitude 1.5-msec rectangular pulse that was recorded as the sound track of the videotape, being dubbed directly onto the sound track so that face and sound were synchronized. The different list types were produced as follows:

No-suffix. J.G. spoke lists of nine single digits at a rate of one digit per second with $15 \mathrm{sec}$ between each list presentation.

Clear auditory speech suffix. After speaking a nine-digit list, J.G. spoke the word "go" in rhythm with the list. A switch control on the amplifier allowed the electrolaryngograph output to be switched off for "go" and natural speech, via a microphone, to be recorded directly onto the videotape sound track. There were no audible indications (a click or cessation of background noise) that this switching had occurred.

Buzz-speech suffix. After speaking a nine-digit list, J.G. spoke the word "go" in thythm with the list, and this was recorded directly onto the videotape sound track.

Silently lipread suffix. After speaking the list, J.G. spoke the word "go" in rhythm with the list, but a switch control on the amplifier allowed the electrolaryngograph output to be switched off for the duration of this item. As in the clear auditory speech suffix condition, no clicks or other signal of a change of input were audible on the videotape to signal that switching had occurred.

Silently lipread lists and sufixxes. To produce the silently lipread list, the master videotape was copied, with the buzz-speech sound track eliminated from the list, but not the suffix, presentation by switching the sound track off for the list items and on again for the suffix. This was done easily and without any audible cues by use of the audiodub control. Because the list items and the suffix were always spoken in rhythm, there was no problem in determining when the list ended and the suffix started. To equate quality of the silent and the buzz-speech tapes, the master tape was copied to produce the buzz-speech tape used in the experiment.

List construction. As in earlier experiments of this type (see, e.g., Campbell \& Dodd, 1982), 15 practice lists similar to those used in the experimental phase of the study and preceding them without a break, were seen to be spoken before the experimental lists were seen. The 48 experimental lists contained 9 digits each. Twelve lists of each of the four suffix conditions occurred in quasirandom order. In addition, 12 10-digit lists were seen, interspersed with the experimental lists, to control for subjects' expecting 9-digit lists and perhaps not looking at the screen when a suffix occurred. Such uncertainty about list length assured that subjects watched the screen until a suffix had been seen to be spoken.

The order of the digits in the 48 experimental lists was determined by randomization, with the constraint that no sequential runs, up or down, greater than two, were permitted. Within these lists, there was no replacement of items (no repetition). However, in the practice lists, a number of items were repeated within a list so that subjects would not use the strategy of predicting the final (ninth) number at the end of a list. The allocation of a particular suffix type to a particular list was also determined by randomization.

Thus, a series of 60 lists of digits was seen by subjects during the experimental phase of this study; 48 of these lists were 9 digits long and were scored for recall accuracy. Suffix type was distributed randomly across these list types. The other lists (10 digits long) were dummy lists and were not scored. A practice series of 159 - digit lists was not scored. The composition of the lists was identical for the buzz-speech and the silent lipreading conditions.

Procedure. The subjects were randomly divided into two groups and taken into one of two rooms, each of which contained a large (22-in.) TV monitor and videotape recorder. One group was shown the silent lipreading tape, the other the buzz-speech tape. The volume setting of the TV monitor was set to a comfortable level for all subjects in the room, which was not soundproof. No independent measure of buzz audibility, of speech signal intensity, or of ambient noise level was taken. (The loudness of the auditory signal may have a bearing on the reliability of these results, and the Discussion section of Experiment 2 refers to this point.)

The subjects, seatei at individual desks, viewed the tapes from a distance of between 2 and $4 \mathrm{~m}$. Instructions for recall were identical for both groups. The subjects were told that they would see someone speaking lists of numbers. They were to watch the speaker and, when she stopped speaking, write down the list that had been said. Sheets were provided for written recall, on which 75 rows, marked into 10 columas, were printed. The subjects were informed to work silently and were told that they had $15 \mathrm{sec}$ for recall. They were instructed not to write down anything while the speaker was speaking and to use strict left-right forward recall, with no backtracking. Where there was uncertainty about what number had been spoken, the subjects were instructed to mark an X, so that the order of the items recalled would be correctly maintained. This was demonstrated by the experimenter, who observed the subjects at all times to ensure that instructions were adhered to. The subjects were also told that some of the lists would be followed by the speaker saying "go." They were to ignore this in their recall. They were further told that some of these last items might sometimes "sound different from the rest of the list."

The experiment lasted $30 \mathrm{~min}$. Afterward, the subjects were told about the purpose of the experiment, and comments were solicited. The subjects in the buzz-speech condition remarked how easy it was to lipread; however, since no subject performed in both conditions, we cannot say whether such ease of lipreading is a reliable feature of the difference between buzz-speech and silent lipreading in this experiment. We have no evidence that the phenomenal impression of "noisy speech" for buzz-speech was consistently achieved or that this affected the rest ${ }^{\top}$ ts. We know from pilot studies that there was no particular moment at which the impression changed from "buzz and lips" to noisy speech. These pilot studies also showed asymptotic recall levels reached within 10 to 12 trials and no abrupt changes in recall accuracy as the experiment proceeded.

\section{Results}

Mean recall accuracy for each serial position (for each suffix type, each condition, and each subject) was calculated, and these data were used for analysis. The effects of list type and of suffix type on general accuracy and on recency are reported in turn.

Recall accuracy. A three-way repeated measures analysis of variance (ANOVA) was performed on mean accuracy/serial position scores. The factors were buzzspeech/silent lipreading (between subjects; the group factor), suffix-type (within subjects), and serial position (within subjects). The four levels of the suffix condition factor were no suffix, silently lipread suffix, buzz-speech suffix, and clear auditory speech suffix.

Mean scores for each condition, for each serial position and each group, are shown in Figure 1. There was no group main effect; buzz-speech and silently lipread speech were equally well recalled under these experimental conditions. 

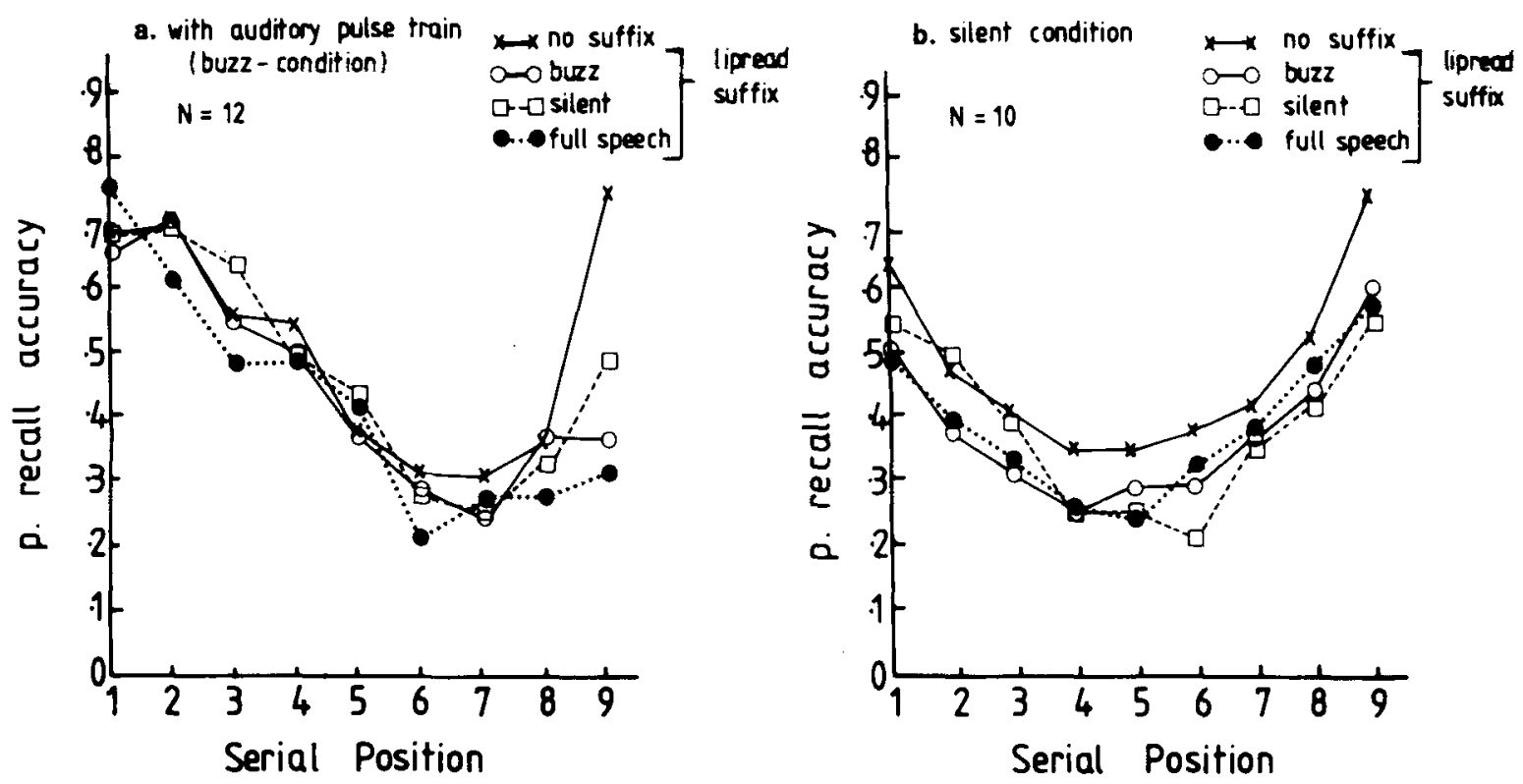

Figure 1. Experiment 1: The mean probability of correct recall for each serial position as a function of sufix type (a) with auditory pulse train accompanying lipreading (buzz), and (b) lipreading alone.

A main effect of suffix type emerged $[F(3,60)=5.82$, $p<.01, M S e=8.93]$. Post hoc tests showed this to be due to a marked advantage for the no-suffix condition over all other conditions. All suffixes impaired recall accuracy, for both buzz-speech and silently lipread speech. There was no interaction between suffix type and group.

A main effect of serial position also was found $[F(8,160)$ $=13.4, p<.001, M S e=16.1]$. Serial position interacted with group $[F(8,60)=5.52, p<.001, M S e=$ 16.1]; the serial recall curves for buzz-speech and for silent lipreading differed in shape, but not in overall accuracy. Post hoc tests showed significant differences at serial positions 4,7 , and 8 . It can be seen from Figure 1 that the silently lipread recall curve is somewhat flatter than the buzz-speech curve; that is, for silently lipread speech, items in the middle of the list were relatively poorly recalled over all positions, whereas for buzzspeech, early items were relatively well recalled, later ones less so.

Second- and third-order interactions were significant in this analysis. The suffix type $\times$ serial position interaction was significant $[F(24,480)=2.58, p<.001, \mathrm{MSe}$ $=2.61]$. Inspection of the figures suggests that recency effects accounted for much of this interaction (the detailed results of an ANOVA for recency effects alone are given below). The groups $\times$ suffix type $\times$ serial position interaction was also significant $[F(24,180)=1.81$, $p<.01, M S \mathrm{e}=2.61$ ]. Again, inspection of the figure suggests that a major theoretically important component of this interaction was in the recency part of the curve (positions 8 and 9), and this component is analyzed further below.

Recency analysis. Since the theoretically important three-way interaction was significant, a second three-way
ANOVA was carried out. In this ANOVA, however, only serial positions 8 and 9 were considered, all other factors remaining as in the earlier analysis.

The results of this ANOVA were as follows: (1) There was no main effect of group at these two serial positions. (2) The main effect of suffix type was significant $[F(3,60)$ $=7.02, p<.001, \mathrm{MSe}=6.84]$ and did not interact with group. Post hoc tests confirmed that, as in the earlier analysis, this effect was due to an advantage of the no-suffix condition over all other suffix types. (3) There was a main effect of serial position $[F(1,20)=80.63, p<.001, M S e$ $=2.23$ ]; that is, overall recency obtained. The effect of the various suffix types on recency can be inferred from the suffix type $\times$ serial position interaction. This was significant $[F(3,60)=9.67, p<.001, M S e=2.01]$, indicating an overall suffix effect. Post hoc testing confirmed that the only significant difference was that between the no-suffix scores at position 8 and each and all suffix scores at this position. (4) There was a significant interaction between group, suffix type, and recency $[F(3,60)=4.02, p<.01, M S e=2.01]$. Post hoc tests are summarized in Table 1 . These tests show that although all suffixes had an equally deleterious effect on recency in the silently lipread condition, there were differentiable suffix effects in the buzz-speech condition. For buzzspeech, recency was most reduced by a clearly heard suffix and a buzz-speech suffix, less so by a silently lipread suffix. Buzz-speech suffix and heard suffix did not have distinguishable effects from each other. A silently lipread suffix had an effect distinguishable from that of no suffix or of a heard suffix, but not from that of a buzz-speech suffix. The best way to characterize these effects is as a gradient of suffix effects. A clear auditory speech suffix had the most marked effect; this was similar to that for 
Table 1

Summary Table of Significance of Post Hoc Tests of Group and Suffix Type on Recency

\begin{tabular}{lcc}
\hline \multicolumn{1}{c}{ Suffix Type Differences } & Buzz-Speech & Silent \\
\hline & Position 8/Position 9 & \\
No Suffix & $p<.01$ & $p<.01$ \\
Buzz Suffix & n.s. & $p<.05$ \\
Silent Suffix & $p<.05$ & $p<.05$ \\
Heard Suffix & n.s. & $p<.05$
\end{tabular}

Position 8 Differences

$$
\text { none none }
$$

Position 9 Differences

\begin{tabular}{lcc} 
No Suffix/Buzz Suffix & $p<.01$ & $p<.02$ \\
No Suffix/Silent Suffix & $p<.02$ & $p<.02$ \\
No Suffix/Heard Suffix & $p<.01$ & $p<.02$ \\
Buzz Suffix/Silent Suffix & $p<.05$ & n.s. \\
Buzz Suffix/Heard Suffix & n.s. & n.s. \\
Heard Suffix/Silent Suffix & $p<.05$ & n.s. \\
\hline
\end{tabular}

a buzz-speech suffix. A lipread suffix had the least marked effect. These analyses also suggest that whereas recency is reduced by all suffix types for each group, the general suffix effect on subjects in the silent lipreading condition was not as pronounced as that on subjects in the buzzspeech condition.

It should be noted that these recency analyses were all performed on positions 8 and 9; we believe (following Balota \& Engle, 1981) that it is in such strict terminal effects that structural rather than attentional recency effects should be sought and explained. Nevertheless, to be sure that these results did not depend on anomalous recall at position 8, we also compared terminal and asymptotic position recall. In Experiment 1 asymptote seems to be around position 6 . Recency analyses contrasting position 6 and position 9 gave results that were not critically different from those reported for position 8 and position 9 . This indicates, at the very least, the robustness of these discriminable recency effects.

\section{Discussion}

The questions posed in the introduction to this report can now be answered. The strict serial recall of lists of single digits presented by lipread speech is not particularly helped by the addition of a synthesized auditory pulse train to signal voiced sound. This is probably because such lists already offer so much contextual help to the speaker/listener that no extra help from the auditory pulse train in disambiguating voiced and voiceless sounds is needed.

Nevertheless, there are differences between the two modes of presentation; buzz-speech generates a sharper serial position function than does silent lipread speech, with the nadir of recall at positions 6 and 7 . Silently lipread speech is relatively more impaired on the earlier serial positions. Buzz-speech appears more closely to resemble auditory list recall. However, the poor recall of the first items of a lipread list may reflect a procedural problem. In recalling silently lipread lists, subjects had no auditory cue that the list was being presented; they were expected to be looking at the screen before the speaker spoke. Despite the long recall time, it is possible that sometimes some subjects did not look at the screen in time to catch the first items of a lipread list. We have observed this in other experiments on silent lipreading. Although this occurred rarely and sporadically, it may have been sufficient to lower mean first-item recall in this condition.

Both list types show marked recency, which, in each case, is impaired by a lipread suffix. However, other features of the suffix have distinguishable effects on buzzspeech lists, but not on silently lipread ones. For silently lipread lists, any lipread suffix impairs recency to the same degree. This is in accordance with earlier findings (e.g., Campbell \& Dodd, 1984). Buzz-lists, however, show a gradient of suffix effects on recency. The most marked effects are those of a buzz-speech suffix and a clearly heard speech suffix; lipreading alone does not interfere much with recency. This gradient is reported in detail for the preterminal/terminal contrast, but it holds also for the asymptote/terminal contrast; it is not due simply to anomalous recall of position 8 items.

Greene and Crowder (1984), on the basis of experiments comparing and contrasting lipread, auditory, and mouthed lists with suffixes of these three types, concluded that structural similarity between list and suffix was the prime determinant of the power of a suffix. If this were the case, in Experiment 1, a buzz-speech suffix should have most impaired recency in a buzz-speech list, and a silently lipread suffix should have most impaired recency for a silently lipread list. This experiment suggests no such crossover pattern of interaction and, therefore, provides no strong support for the structural-similarity hypothesis in determining the extent of recency and suffix effects. What the results of the present experiment do suggest, rather, is that auditory lists show recency and suffix effects that can be affected by perceived structural similarity, even when the auditory input alone is insufficient to generate effective phonetic perception. In contrast, silently lipread lists are susceptible to all or any speech event in reducing recency.

A number of experiments have now demonstrated that the speechlikeness of an auditory event can be contextually controlled and that such contextual controls, in turn, can determine the extent to which suffixes can impair recency. The first and most telling of such demonstrations was that of Ayres, Jonides, Reitman, Egan, and Howard (1979). They showed that an ambiguous /wa/ sound could have a suffix effect if subjects were informed that it was the syllable "wah," but not when it was presented as "a trumpet note." Further demonstrations of such expectations on modality-specific effects are described in the General Discussion. In Experiment 2 a rather different interpretation of the present findings was tested. 


\section{EXPERIMENT 2}

Experiment 1 showed that recency for a silently lipread list was affected equally by three types of lipread suffixes, two of which were synchronized with heard sounds and one of which was not. By contrast, the lipread lists accompanied by a heard buzz showed differential suffix effects; in particular, the silently lipread suffix interfered least, the fully auditorily specified suffix interfered most.

Experiment 1 can be formally compared with, and considered structurally equivalent to, an auditory list and suffix experiment reported by Routh and Lifschutz (1975). They examined the effect of adding an irrelevant tone to each spoken item of the auditorily presented list and/or to the heard suffix. They demonstrated that adding the tone affected neither overall accuracy of recall nor auditory recency. However, the effect of a suffix varied systematically as a function of the presence or absence of the tone in the list and in the suffix. When each list item was accompanied by a tone, the suffix was effective only if it, too, carried a tone. When the list had no accompanying tone for each spoken item, it was immaterial whether the suffix was accompanied by a tone; recency was similarly reduced in each case. Routh and Lifshutz redescribed this asymmetrical effect as follows: adding an extraneous auditory feature to the suffix did not materially alter its power to reduce heard recency; however, subtracting a feature from the suffix, when that feature had been present in the list, reduced the suffix effect. Two things should be noted about this. First, the tone feature was extraneous to categorical perception of the heard items; it neither aided nor impaired it. Second, examination of Routh and Lifshutz's data suggests that the suffix effects extended into preterminal positions in recall. As Balota and Engle (1981) pointed out, the effects of a suffix on preterminal recall appear to have a basis in attentional factors that are often quite specific to the task. It is likely, therefore, that Routh and Lifshutz's tone feature suffix effects reflect, in part, an attentional component to the recall task.

In Experiment 1 reported here, we saw that the silently lipread condition, like the auditory-without-tone condition of Routh and Lifschutz (1975), showed recency that was disrupted by suffixes that were similar to, and also that contained more information than, the list. In the buzzspeech condition, as in Routh and Lifschutz's study, the effectiveness of the suffix was reflected by the loss of a feature; in our case, the silently lipread suffix failed to eliminate recency as thoroughly as did the buzz or the auditorily specified suffix. (The extra, differentiated, suffix effect of a fully specified auditory speech suffix, however, might not fit so comfortably within Routh and Lifschutz's framework unless we make the additional assumption that the effect can comprise multiple added features that have an additive effect. This seems a singularly unmotivated assumption. However, it is not entirely clear, from the single demonstration of Experiment 1, to what extent a full speech suffix does have an effect over and above the buzz-speech suffix.)
An alternative explanation for the findings of Experiment 1 is that they reflect what we interpret as an attentional type of effect in Routh and Lifschutz's (1975) study for silently lipread lists. Experiment 2 was designed to distinguish between the two interpretations of the differential suffix effect of Experiment 1. Recall in the buzz-speech condition (identical to that of Experiment 1) was contrasted with recall of an identical list to which a pure tone was added (buzz-and-beep). If the suffix types are not given an additional tone feature, according to Routh and Lifschutz's theory, recency should survive any such suffix unscathed. At the very least, a reduced and undifferentiated suffix effect of such "unbeeped" suffixes on a lipread buzzand-beep list should occur. By contrast, the speechlikeness hypothesis predicts similar effects of lipread, buzz-speech, and full auditory suffixes for a buzz-and-beep list as for a buzz-speech list without the tone. Adding the tone to the list should neither improve nor impair the speech quality of the list and its concomitant suffix effects.

\section{Method}

Stimulus preparation. Two copies were made of the buzz-speech videotape used in Experiment 1. One copy was used for the buzzspeech condition. To the second tape, however, a pure tone signal of $1000 \mathrm{~Hz}$, lasting $250 \mathrm{msec}$, was added to each spoken list number and in synchrony with it. The tone and its duration were produced and controlled by a WAVETEK tone generator attached to a BRND logic board. These bursts of tone were dubbed onto the buzz-speech videotape. The experimenter timed each burst to coincide with the onset of each spoken digit. In this way, a videotape was produced in which buzz-speech was seen and heard for the digit lists, but to which a pure tone signal had been added. The suffix conditions remained as in the buzz-speech condition of Experiment 1 . The pitch, duration, and volume characteristics of the tone were as similar as possible to those described by Routh and Lifschutz (1975). Apart from the addition of tone to the lists, the sound and vision qualities of the buzz-speech and the buzz-and-beep videotapes were indistinguishable.

Procedure. Two groups of undergraduate subjects (in this case, undergraduates of Oxford University) were run on the buzz-speech or the buzz-and-beep speech condition. There were 12 subjects in each group. All other experimental conditions and instructions were as in Experiment 1.

\section{Results}

Figure 2 shows the mean accuracy for each serial position for each condition. An ANOVA, with condition (buzz vs. buzz-and-beep) as the between-subjects factor and serial position (9 levels) and suffix type (4 levels) as the two within-subjects factors, was performed. This showed no significant effect of condition (buzz vs. buzzand-beep) overall $(F=0.09)$. Condition interacted significantly both with serial position $[F(8,176)=2.04, p<$ $.05, M S \mathrm{e}=0.57]$ and with suffix type $[F(3,66)=7.71$, $p<.01, M S e=0.35]$. A further significant interaction was between suffix type and serial position $[F(24,528)$ $=2.10, p<.01, M S e=1.29$ ].

Inspection suggests, and post hoc tests confirmed, that the theoretically significant interactions concerned recency in the two experimental conditions. Overall, buzz-speech differed from buzz-and-beep in showing extensive recency (in fact, this is confined to the no-suffix condition, and 
a. auditory pulse train (buzz condition)

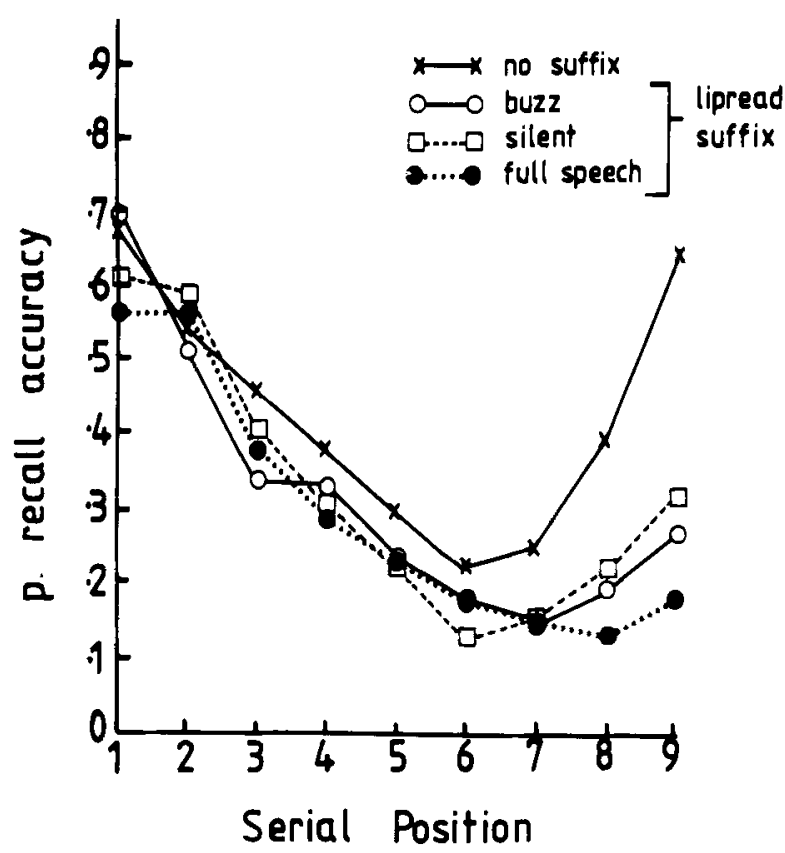

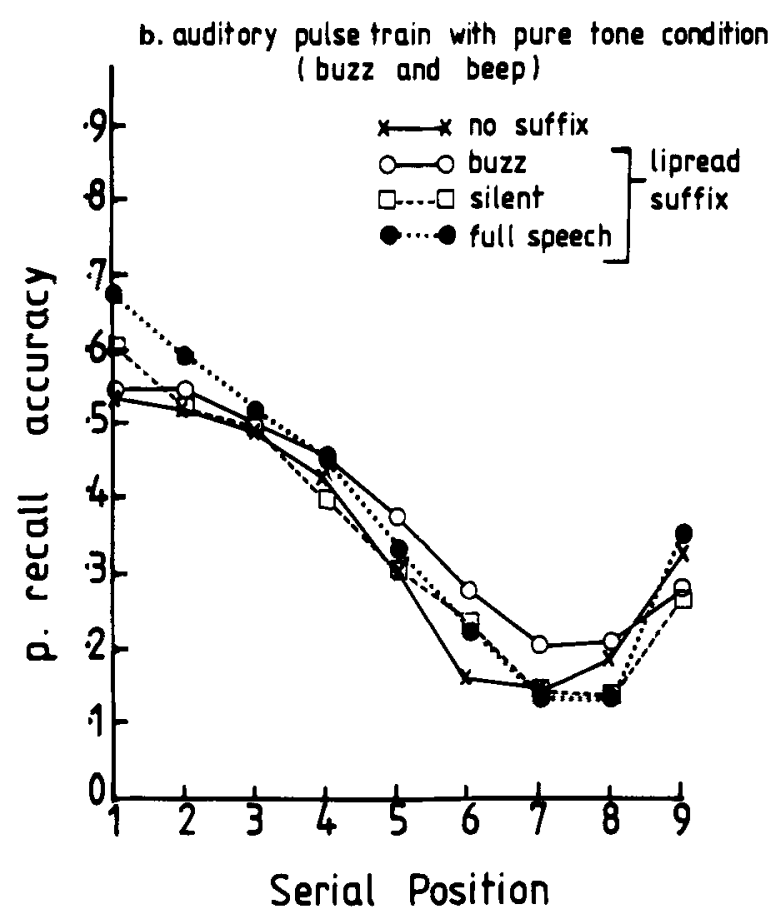

Figure 2. Experiment 2: The mean probability of correct recall as a function of serial position for each suffix type (a) with auditory pulse train accompanying lipreading (buzz), and (b) with auditory pulse train and pure tone (buzz-and-beep).

is reduced by all suffix types). Buzz-and-beep lists, in contrast, showed little recency in the no-suffix condition (a $t$ test on the position 8-position 9 difference was not significant) and no difference between suffix and no-suffix conditions in this respect. Separate analyses of the two presentation conditions gave a finer grained picture of these differences.

Buzz-speech ANOVA. A two-way ANOVA of performance of the 12 subjects in the buzz-speech condition, with serial position and suffix type as the two factors, confirmed a significant effect of suffix type $[F(3,33)=7.8$, $p<.01, M S \mathrm{e}=4.03]$ and of serial position $[F(8,88)=$ $22.9, p<.001, M S e=5.51]$ and a significant interaction between these factors $[F(24,264)=2.2, p<.01, M S \mathrm{e}$ $=1.41]$. Among the significant post hoc differences were those of the no-suffix condition and the suffix conditions at positions 8 and 9 . There was a significant difference at position 9 between the effect of a full-speech suffix and that of buzz-speech $(p<.02)$. This confirms and replicates, at least in part, the differential suffix effects for buzz-speech noted in Experiment 1.

Buzz-and-beep ANOVA. A similar two-way ANOVA was performed on the results of the 12 subjects in the buzz-and-beep condition. There was no main effect of suffix type $(F=.207)$ and no interaction between condition and serial position $(F=1.19)$; the serial position effect was significant $[F(8,88)=23.12, p<.01, M S e=$ 5.63]. As can be seen in Figure 2b, the lack of an interaction is due primarily to the absence of a substantial recency effect when there was no suffix.

\section{Replication}

This failure to find a recency effect for the buzz-andbeep condition when no suffix was present, in the face of overall similarity of recall between the buzz and the buzz-and-beep conditions, was unexpected. Thus, a replication of the buzz-and-beep condition was performed, using 22 new subjects from the University of London. In this study, identical procedures were used to run the volunteer subjects as were used in Experiment 2.

Table 2 shows means and standard deviations for each serial position for each condition in this replication. A two-way ANOVA on these results confirmed, in general form, the findings on the 12 subjects of Experiment 2 . That is, there was a significant effect of serial position, but no significant suffix-type effect and no interaction between suffix type and serial position.

The last two serial positions were inspected separately, in case any recency effects were hidden in the general analysis. Although there was a slight recency effect for the no-suffix condition in this replication (a $t$ test on position 8-position 9 difference was significant at $p=$ $.05)$, the difference between suffix and no-suffix conditions was not significant.

\section{Discussion}

Buzz-speech effects. One of the positive results of Experiment 2 was that it partially confirmed the differential suffix effects on buzz-speech recency that emerged from Experiment 1. A full, auditorily specified heard-andseen speech suffix reduced recency more effectively than 
Table 2

\begin{tabular}{|c|c|c|c|c|c|c|c|c|}
\hline \multirow{2}{*}{$\begin{array}{c}\text { Serial Position } \\
\text { Accuracy }\end{array}$} & \multicolumn{2}{|c|}{ No Suffix } & \multicolumn{2}{|c|}{$\begin{array}{l}\text { Silent Lipread } \\
\text { Suffix } \\
\end{array}$} & \multicolumn{2}{|c|}{$\begin{array}{c}\text { Buzz-Speech } \\
\text { Suffix } \\
\end{array}$} & \multicolumn{2}{|c|}{$\begin{array}{l}\text { Full Speech } \\
\text { Suffix }\end{array}$} \\
\hline & $M$ & $S D$ & $M$ & $S D$ & $M$ & $S D$ & $M$ & $S D$ \\
\hline 1 & 56.4 & 25.9 & 63.0 & 23.9 & 53.4 & 26.1 & 60.5 & 19.7 \\
\hline 2 & 59.7 & 25.2 & 62.5 & 23.3 & 48.5 & 20.0 & 53.8 & 23.7 \\
\hline 3 & 59.0 & 24.7 & 52.3 & 23.1 & 49.0 & 16.2 & 51.7 & 19.4 \\
\hline 4 & 46.4 & 21.6 & 43.9 & 18.3 & 47.2 & 19.0 & 47.0 & 18.6 \\
\hline 5 & 40.6 & 26.2 & 36.1 & 19.5 & 35.9 & 17.3 & 39.6 & 18.5 \\
\hline 6 & 31.0 & 15.3 & 23.8 & 13.2 & 31.3 & 18.0 & 29.0 & 18.2 \\
\hline 7 & 20.9 & 14.9 & 17.6 & 12.2 & 20.5 & 14.5 & 19.3 & 12.9 \\
\hline 8 & 20.1 & 15.1 & 18.6 & 12.0 & 19.4 & 16.0 & 14.1 & 14.4 \\
\hline 9 & 35.1 & 20.8 & 27.1 & 20.4 & 28.6 & 14.65 & 25.9 & 18.7 \\
\hline
\end{tabular}

did a sensorily similar buzz-speech suffix or a silently lipread suffix. This finding offers further support for the notion that speech quality, rather than sensory similarity, may determine the effectiveness of some suffixes on lists that are seen to be spoken. Nevertheless, the replication of the suffix gradient for buzz-speech recency is not absolute. Although the ordering of suffix effectiveness was the same in the two experiments, the significant differences were not. In particular, in Experiment 2 all suffixes were more effective than in Experiment 1. One reason for this discrepancy may be that the relative loudness of the buzz-speech was not controlled adequately across the two studies. It is our impression that the quieter the auditory buzz, the more speechlike the stimulus seems to be. Thus we suspect that in Experiment 2 the TV volume setting was lower for the buzz-speech condition than in Experiment 1. There also may have been more ambient noise. Alternatively, subject variability may underlie this discrepancy (overall recall in Experiment 2 was poorer than in Experiment 1). We plan systematic studies on the effect of buzz loudness on recall.

In an exhaustive study manipulating the acoustic properties of suffixes on natural auditory list recall, Morton et al. (1981) showed that synthesized speech sounds, as well as filtered natural speech sounds, produced effective suffix impairments of recency. However, when such acoustic manipulations were combined to reduce the speechlikeness of the suffix, the suffix effect was often lost. The effectiveness of an artificial suffix was not determined by subjects' ratings of speechlikeness for these items, a finding that led these authors to claim that subjective and acoustic influences do not interact to produce a suffix effect: The acoustic and the subjective qualities of speechlikeness differ, and the acoustic determinants of speechlikeness, alone, produce the standard type of recency and suffix effect.

There is a sense in which these buzz-speech studies can be viewed as part of the general demonstration that speechlikeness, rather than the purely acoustic properties of list and suffix, determines recency and suffix effects. However, the studies advance the demonstration in some important respects. First, they provide the only demon- stration that audiovisual material that cannot acoustically lead to the perception of speech can generate auditoryseeming recency. Second, such speechlike recency is affected differentially by different suffix types; in particular, a clearly heard auditory suffix has at least as marked an effect on buzz-speech recency as does a buzz-speech suffix. Third, the differential effect of these suffixes seems to follow the graded pattern of that shown to date only for natural auditory lists and not that for silent lipread lists. Since this difference emerges when the acoustic properties of the heard list are specified in a manner that cannot support perception and recall on its own, they cannot be due to the acoustic properties of speechlikeness but, by contrast, seem to reflect something else. These properties may or may not have anything to do with subjects' judgments of good speech or phonemic qualtiy. Whether they are connected with changes in the phenomenal impression of buzz-speech during the period of the experiment is also an open question, and one for further research to answer.

Buzz-and-beep condition. The surprising finding of Experiment 2 is that adding a tone to the lipread buzzspeech list did not leave the recency function intact, as it did when added to a fully auditorily specified list (Routh \& Lifschutz, 1975). Instead, the buzz-and-beep list showed a recall function remarkably similar to that seen for written lists (e.g., Hitch, 1975; Morton, 1978), with very little recency and with no very marked terminal suffix effects of any sort. This general picture held for the main experiment, in which buzz-speech and buzz-and-beep speech were contrasted and in the replication of buzz-andbeep alone. Any recency effects for this condition must be small indeed, if they do not reliably emerge in an investigation of 34 subjects.

Although this pattern of suffix effects might be consistent with Routh and Lifschutz's (1975) feature-based explanation, we would claim that the failure to find recency in this condition means that Routh and Lifschutz's model may not be appropriate in this case. The discovery that adding a tone to a buzz-speech list fails to produce similar recency to that for a buzz-speech list alone suggests some very different mechanisms to explain lipread (with 
or without ancillary auditory inputs) list recall and auditory list recall. This distinction is the hub of the General Discussion.

\section{GENERAL DISCUSSION}

The classical finding in immediate list recall is that heard lists show recency and seen lists do not. In these two experiments, we used a variety of modality presentation conditions that cannot fit this description. In the first place, a silently lipread list was shown, yet again, to generate recency that was overwritten by a heard or lipread suffix. In the second place, a lipread list that had complementary speech-feature information added, in the form of a synchronous auditory pulse train signaling voice-pitch contour, produced recency that was, if anything, even more speechlike than silent lipreading. Such buzz-speech list recency is overwritten most effectively by a fully specified auditory speech suffix. This suggests that sensory similarity of suffix and list is not a necessary determinant of recency and suffix effects where they occur. But the finding for a buzz-speech list with a pure tone accompanying each spoken item (buzz-and-beep) is most surprising: a heard list, comprising auditory features that are both useful and redundant, failed to engender recency, whereas seen (lipread) lists did.

It is important to establish what the addition of the tone to the buzz-speech input did not do. It did not materially impede the processing of the stimulus as a digit to be recalled in writing, for there was no sign of any overall recall decrement for buzz-and-beep lists compared with buzz-speech lists. Where, then, did the tone have an effect? Our provisional explanation is necessarily post hoc. Good recall of the last items of an ordered number or letter list reflects a second look at a level of representation, that is, a trace of the input or of processes that have been performed upon it. Such a trace must necessarily support identification of the stimulus too; its effect in immediate recall is, in this sense, an "echo" of its identification function. The primary reason this representation persists in time is that phonemes are often not identified until after the registration of a sensory speech event; that is, context effects in auditory speech perception typically can work backward in time, as well as predictively (see, e.g., McClelland \& Elman, 1986).

The trace need not be sensory in the strict modality sense; indeed, we have argued elsewhere that the usable trace for silently lipread material is a phonetic, not a strictly sensory, representation of the stimulus configuration (Campbell, 1987). Such phonetic traces cannot usually be derived directly from written material, except under special conditions (see Campbell, 1987, Experiment 2; Crowder, 1983). Lipreading gives rise to a phonetic percept and therefore leaves an accessible phonetic trace. This may, however, be underspecified with respect to every phonetic component of the identified speech sound, because context rather than feature information will have determined identification. Auditory speech inputs automatically give rise to phonetic representations that are the main source of auditory recency and suffix effects. It is likely that the more fully specified, phonetically, the sensory input to the phonetic trace is, the more powerful its effects in and on recency. Hence, auditory suffix effects can be more extreme for lipread lists than are (sensorily similar) lipread suffixes (although they were not in Experiment 1), and lipread suffixes, although they certainly affect auditory recency, are not always as effective as auditory ones (Greene \& Crowder, 1984; Spoehr \& Corin, 1978).

The auditory input does not have to be speech in order to utilize phonetic processing. This is the case for buzzspeech, and it accounts for the congruence of phenomenal impression and auditory recency in buzz-speech recall and for the relatively greater effect of a heard suffix than the other suffix types in Experiments 1 and 2. Phonetic processing of ambiguous auditory stimuli can be under strategic control, as the "wah" experiment of Ayres et al. (1979) showed. But when the auditory input is unambiguously speech, phonetic processing cannot be switched off. (Auditory suffix effects are not strategically manipulable; see Hitch, 1975.)

When the auditory input is fully specified, as in Routh and Lifschutz's (1975) study, adding an additional tone cannot further affect the phonetic processor; the spoken number is already fully phonetically specified. Under these conditions, the extra stimulus dimension-the tone that accompanies the presentation of each list itemfunctions as an attentional or grouping organizer. Thus, if the suffix is without a tone, it is less effective than a tone-accompanied suffix in reducing recall in toneaccompanied spoken lists. By contrast, if the list items are not accompanied by tones, any type of speech suffix (with or without tone) will be equally effective. As an indication that the effects described by Routh and Lifschutz are at least partly attentional in origin, it should be noted that their asymmetrical suffix effects stretch back into the preterminal parts of the list. As we have remarked elsewhere in this paper, such preterminal suffix effects implicate attentional or grouping processes. Routh and Lifschutz's effects were not specific to the last item(s) of the lists. It is for this reason, a reason additional to phonetically determined recency, that the suffix effects they describe are asymmetrical, with the absence of a tone feature failing to function as an attentional organizer.

In Experiment 2, however, the stimulus array did not provide a full phonetic specification of the item seen to be spoken. Lipreading provides some of the features of place of articulation, and buzz provides voice-pitch contour, but nevertheless the stimulus cannot provide all the phonetic features needed for identification. In these conditions, the phonetic processor is still effectively open to all auditory "possibilities" and attempts to process the tone as part of the speech percept. This need not impair identification, yet it may mean that any transient representation of the activation pattern that supports identification may not be sufficiently clearly specified to support the 
"second look" that recency reflects. It is because the irrelevant tone was being processed as part of the identification of the spoken number that it persisted, in such a deleterious fashion, when the last items were recalled.

This adventitious result may, by prompting these theoretical speculations, allow us to start to develop new insights into the puzzles of recency and suffix effects in immediate memory. There are clearly a multiplicity of reasons for recency in heard and some seen lists. The patterns of interference between such recency effects and putative suffixes clarify the source of the last-item advantage. The present study indicates that sensory similarity of list and suffix may not always determine recency and suffix effects and that not all lipread-with-sound lists show recency in immediate recall. In particular, where phonetic identification (from lipread, buzz-speech, or buzz-andbeep speech) leaves a trace on which recency can be based, the recency and suffix effects that emerge reflect in large part how well this trace has been specified from the input.

\section{REFERENCES}

Ayres, T. J., Jonides, J., Reitman, J. S., Egan, J. C., \& Howard, D. A. (1979). Differing suffix effects for the same physical suffix. Journal of Experimental Psychology: Human Learning \& Memory, 5, 315-321.

Balota, D. A., \& ENGLE, R. (1981). Structural and strategic effects in the stimulus suffix effect. Journal of Verbal Learning \& Verbal Behavior, 20, 346-357.

CAMPBELL, R. (1987). Common processes in immediate memory. In D. A. Allport, D. MacKay, W. Prinz, \& E. Scheerer (Eds.), Language perception and production: Common mechanisms in listening, speaking, reading, and writing (pp. 131-149). NY: Academic Press.

CAMPBell, R., \& DodD, B. (1980). Hearing by eye. Quarterly Journal of Experimental Psychology, 32, 85-99.

CAMPBell, R., \& DodD, B. (1982). Some suffix effects on lipread lists. Canadian Journal of Psychology, 36, 509-515.

CAMPBell, R., \& DoDD, B. (1984). Aspects of hearing by eye. In H. Bouma \& D. G. Bouwhuis (Eds.), Attention and performance $X$ (pp. 300-311). Hillsdale, NJ: Erlbaum.
Crowder, R. G. (1983). The purity of auditory memory. Philosophical Transactions of the Royal Society of London, 302B, 251-265.

Crowder, R. G., Morton, J. (1969). Precategorical acoustic storage (PAS). Perception \& Psychophysics, 5, 365-373.

GATHERCOLE, S. E. (1987). Lipreading: Implications for theories of short-term memory. In B. Dodd \& R. Campbell (Eds.), Hearing by eye: The psychology of lipreading (pp. 228-240). London: Erlbaum.

Greenberg, S., \& ENGle, R. W. (1983). Voice change in the stimulus suffix effect: Are the effects structural or strategic? Memory \& Cognition, 11, 551-556.

Greene, R. L., \& Crowder, R. G. (1984). Modality and suffix effects in the absence of auditory stimulation. Journal of Verbal Leaming \& Verbal Behavior, 23, 371-382.

Hitch, G. (1975). The role of attention in the visual and auditory suffix effect. Memory \& Cognition, 3, 501-505.

McClelland, J. L., \& Elman, J. L. (1986). The TRACE model of speech perception. Cognitive Psychology, 18, 1-86.

Morton, J. (1978). Perception to memory. Cognitive Psychology: Open University Social Sciences, a third level course (Unit 14). Milton Keynes, England: The Open University.

Morton, J., Crowder, R. G., \& Prussin, H. A. (1971). Experiments with the stimulus suffix effect. Journal of Experimental Psychology, 91, 169-190.

Morton, J., Marcus, S. M., \& OtTley, P. (1981). The acoustic correlate of "speechlike": A use of the suffix effect. Joumal of Experimental Psychology, General, 110, 568-593.

NAIRNE, J. S., \& Walters, V. L. (1983). Silent mouthing produces modality- and suffix-like effects. Journal of Verbal Learning \& Verbal Behavior, 22, 475-483.

Rosen, S. M., Fourcin, A. J., \& Moore, B. C. J. (1981, Summer). Lipreading connected discourse with fundamental frequency information. British Society of Audiology Newsletter, pp. 42-43.

Routh, D. A., Lifschutz, A. J. (1975). An asymmetrical effect of similarity in the attenuation of stimulus suffix interference. Journal of Verbal Learning \& Verbal Behavior, 14, 95-104.

Spoenr, K. T., \& CoRIN, W. J. (1978). The stimulus suffix effect as a memory coding phenomenon. Memory \& Cognition, 6, 583-589.

\section{NOTE}

1. The effects of silent mouthing (Greene \& Crowder, 1984; Nairne \& Walters, 1983) are not considered here.

(Manuscript received October 16, 1986; revision accepted for publication October $18,1987$. 